\title{
OCORRÊNCIA, DANOS E CONTROLE DE ÁCARO-BRANCO Polyphagotarsonemus latus (BANKS, 1904) (ACARINA: TARSONEMIDAE) EM CULTIVO PROTEGIDO DE PIMENTÃo
}

\author{
Occurrence, damage and control of the Mite \\ Polyphagotarsonemus latus (Banks, 1904) (Acarina: \\ Tarsonemidae) on Sweet Pepper Cultivated in Greenhouses
}

\author{
Paulo Roberto Valle da Silva Pereira \\ Entomologista, Doutor - Embrapa Trigo, Cidade - Estado,. E-mail: paulo@cnpt.embrapa.br. \\ Bernardo de Almeida Halfeld-Vieira \\ Fitopalogista, Doutor - Embrapa Roraima, Boa Vista - RR. E-mail: halfeld@cpafrr.embrapa.br. \\ Kátia de Lima Nechet \\ Fitopalogista, Doutor - Embrapa Roraima, Boa Vista - RR. E-mail: katia@cpafrr.embrapa.br. \\ Moisés Mourão Júnior \\ Bioestatístico, Mestre - Embrapa Roraima, Boa Vista - RR. E-mail: mmourao@cpafrr.embrapa.br.
}

\begin{abstract}
Resumo
O cultivo protegido de pimentão vem se tornando uma alternativa vantajosa para os produtores devido à redução das perdas e o aumento da produtividade, além de apresentar vantagens em relação ao cultivo a campo. Dentre as pragas que atacam o pimentão, destaca-se o ácaro-branco Polyphagotarsonemus latus, cujos danos são severos e podem reduzir drasticamente a quantidade e qualidade dos frutos produzidos. Em experimento realizado em casa de vegetação foi avaliada, em cinco híbridos de pimentão, a eficácia do ingrediente ativo abamectina, na dose de 1,8 g / 100 1 d'água, no controle deste ácaro. A avaliação baseou-se nos parâmetros: plantas atacadas (\%); plantas não atacadas (\%) e plantas recuperadas (\%). As pulverizações foram realizadas aos 40, 60 e 80 dias e as avaliações aos 45, 65 e 85 dias após o transplante. Houve diferença estatística significativa entre os híbridos testados, quando comparados entre si ou em diferentes datas de avaliação. O híbrido Nathalie apresentou o menor percentual de plantas atacadas em todas as avaliações, com uma variação de 13 a 28\% das plantas. O híbrido Martha apresentou o maior percentual de plantas atacadas, com uma variação de 38 a 59\% das plantas.
\end{abstract}

Palavras-chave: Polyphagotarsonemus latus; Pimentão; Cultivo protegido. 


\begin{abstract}
The cultivation of sweet pepper in greenhouses is becoming an advantageous alternative to growers due to loss reduction, increase in productivity and also by showing advantages in relation to field cultivation. Among the pests that attack sweet pepper, the mite Polyphagotarsonemus latus is distinguished by the severe damage that it can cause, leading to a drastic reduction in the amount and quality of fruit production. In a greenhouse experiment, using five hybrids, the efficacy of abamectin, in the dosage of $1.8 \mathrm{~g} / 100$ lof water, was evaluated to control this mite. The evaluations were based on the following parameters: attacked plants (\%); not attacked plants (\%) and recovered plants (\%). The spraying were performed at 40, 60 and 80 days and evaluations conducted at 45, 65 and 85 days after transplantation. There were significant statistical differences between hybrids when compared among them or in different evaluation dates. The hybrid Nathalie presented the lesser percentage of attacked plants in all evaluations, varying from 13 to $28 \%$ of the plants. The hybrid Martha presented the bigger percentage of attacked plants, varying from 38 to $59 \%$ of the plants.
\end{abstract}

Keywords: Polyphagotarsonemus latus; Sweet pepper; Greenhouse.

\title{
INTRODUÇÃO
}

O pimentão (Capsicum annuum L.) pertence à família das solanáceas e está entre as cinco hortaliças com maior área cultivada no Brasil e no mundo. Nos grandes centros urbanos do país, o volume de comercialização é bastante expressivo. Segundo dados da CEAGESP no ano de 2001, o volume total comercializado foi de 39.634 toneladas (FNP, 2003). No estado de Roraima, até 1998, a produção do pimentão não atendia nem o mercado consumidor local, necessitando, no ano de 1995, no período de janeiro a setembro, da importação da Venezuela de 42 toneladas da hortaliça (OLIVEIRA; LUZ, 1998).

O cultivo protegido de hortaliças vem se tornando uma alternativa vantajosa para produtores que a utilizam para aumentar lucros, devido à redução das perdas e aumento da produtividade, além de apresentar vantagens em relação ao cultivo a campo (LORENTZ et al., 2002). Segundo Serrano Cermeño (1990), o pimentão é uma das culturas mais indicadas para cultivo protegido, pela grande produtividade atingida que pode variar de 80 a 150 t.ha ${ }^{-1}$.

As cultivares híbridas apresentam algumas vantagens sobre as demais, com melhores respostas às exigências do produtor e do mercado, como: alto potencial produtivo (rendimento e qualidade); maior adaptação aos sistemas de cultivo; produção de frutos de maior peso médio e resistência às principais doenças da cultura (NASCIMENTO et al. 2002). Em estudos realizados com a cultura de pimentão sob cultivo protegido e orgânico, Nanneti et al. (2002) observaram que os híbridos Magali-R e Furtuna Super apresentaram melhor desempenho em relação à quantidade de frutos/planta e maior peso fresco de fruto/planta.

Entretanto, a cultura do pimentão é suscetível ao ataque de alguns artrópodos-praga que não só aumentam o custo de produção da cultura devido ao uso de produtos para controlá-los, como também podem ocasionar perdas significativas na produção. Dentre eles, destaca-se o ácaro-branco Polyphagotarsonemus latus (Aracnida: Acari: Tarsonemidae), cujos danos são muito severos e podem reduzir drasticamente a quantidade e qualidade dos frutos produzidos. Esse ácaro é polífago e cosmopolita, sendo conhecido também como ácaro-tropical, ácaro-da-rasgadura e ácaro-da-queda-do-chapéu-domamoeiro. O ácaro-branco é de difícil visualização a olho nu e durante o seu desenvolvimento passa pelos estágios de ovo, larva, "pupa" e adulto. Os ovos medem aproximadamente 0,1 mm de comprimento, apresentam coloração perolada, com linhas de manchas brancas na superfície e são colocados de forma isolada na face inferior das folhas (FLECHTMANN, 2000; GALLO et al., 2002). O período de incubação varia de 1 a 3 dias, a $27^{\circ} \mathrm{C}$, e dos ovos eclodem larvas que possuem três pares de pernas, coloração esbranquiçada e, quando completamente desenvolvidas, medem cerca de $0,15 \mathrm{~mm}$ de comprimento. O 
estágio larval dura em média dois dias quando a temperatura média é de $27^{\circ} \mathrm{C}$. As larvas, quando completamente desenvolvidas, permanecem em repouso, dentro de um invólucro originado do tegumento e que se afila para as duas extremidades. Dentro desse invólucro ocorre a formação do quarto par de pernas e, após esta fase, que é imóvel e referida como "pupal", emerge o adulto. Nessa fase, os indivíduos ficam em repouso por 1 a 2 dias (FLECHTMANN, 2000).

A fêmea mede aproximadamente $0,17 \mathrm{~mm}$ de comprimento por 0,11 de largura, tem coloração amarela brilhante e quatro pares de pernas. As pernas do quarto par são pouco usadas para locomoção, sendo reduzidas e terminando em duas longas cerdas. O macho é menor, com cerca de $0,14 \mathrm{~mm}$ de comprimento por 0,08 mm de largura, de coloração branca hialina e possui o quarto par de pernas muito avantajado e em forma de clava como apresenta a Figura 1, o que lhe permite carregar a pupa da fêmea por algumas horas antes de se transformarem em adultas, de forma que no momento da emergência a cópula seja garantida. Esse hábito auxilia a disseminação e concorre para garantir a propagação da espécie (FLECHTMANN, 2000; GALLO et al., 2002). Em trabalho de Soroker et al. (2003), é observada outra maneira de disseminação de $P$. latus, por intermédio de moscas brancas, que levam estes ácaros para outras plantas, aderidos na cera que cobre seus corpos.

O ácaro-branco procura evitar a luz direta, mantendo-se na superfície inferior das folhas da

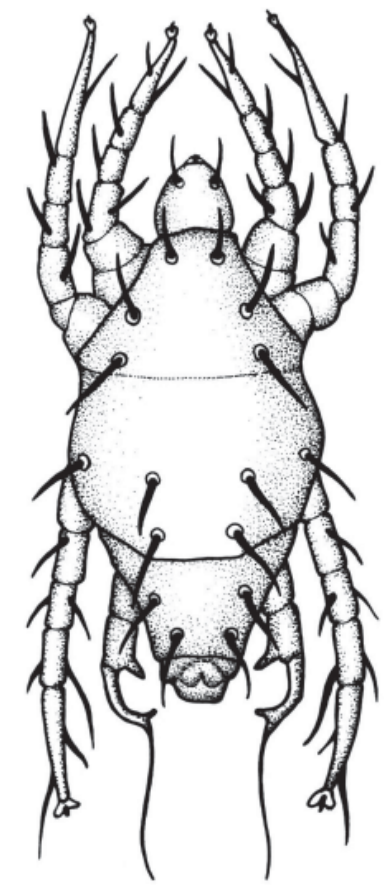

(A)

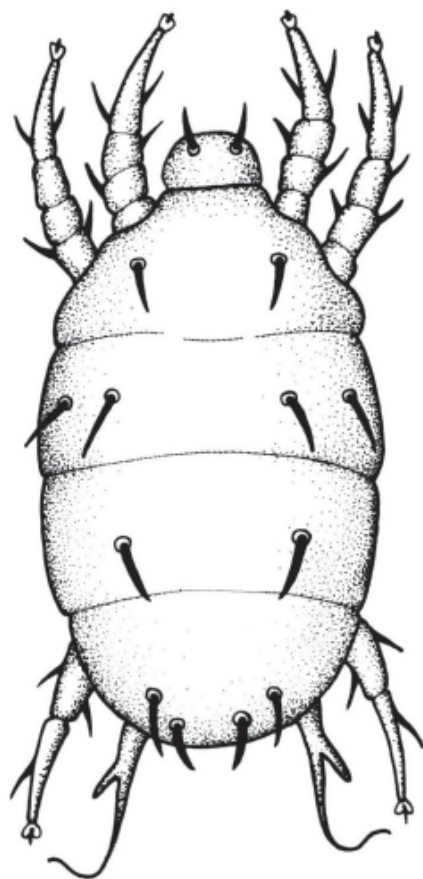

(B)

FIGURA 1 - Ácaro-branco Polyphagotarsonemus latus (Banks, 1904) (Acarina:

Tarsonemidae): A) macho; B) fêmea

Figure 1 - Mite-white Polyphagotarsonemus latus (Banks, 1904)(Acarina: Tarsonemidae): A) male; B) female

planta atacada, mostrando preferência pelas folhas do ponteiro. As plantas atacadas apresentam sintoma característico, com as folhas deformadas, enrugadas para baixo ou com enrugamento da nervura principal e com o pecíolo alongado como apresenta a Figura 2. 


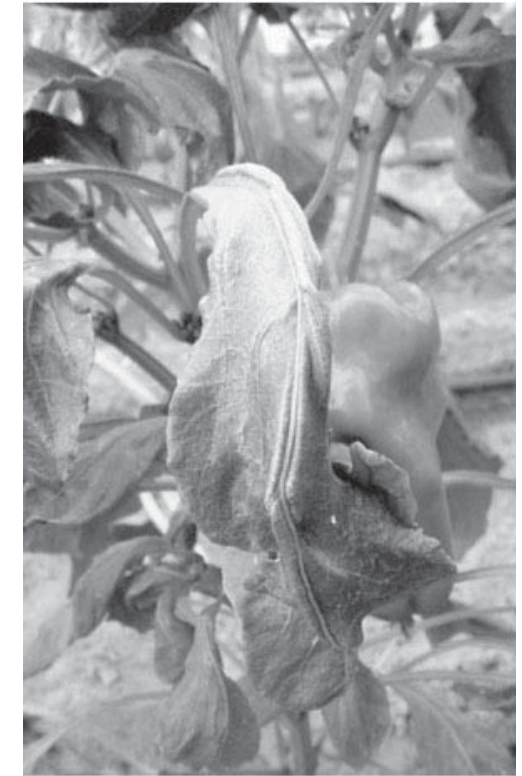

A)

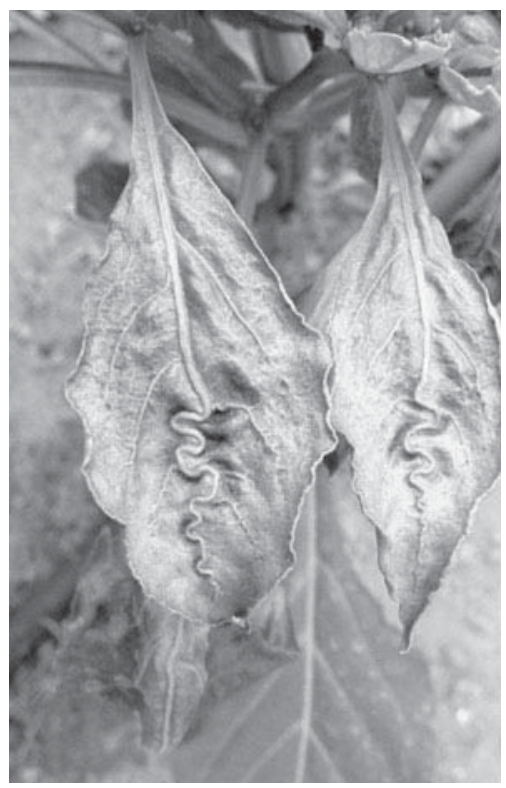

B)

FIGURA 2 - Danos causados em folhas de pimentão pelo ataque de Polyphagotarsonemus latus (Banks, 1904) (Acarina: Tarsonemidae): A) folhas enrugadas para baixo e com pecíolo alongado; B) enrugamento da nervura principal (Fotos: Bernardo A. Halfeld-Vieira)

Figure 2 - Actual damages in sweet pepper leves for the attack of Polyphagotarsonemus latus (Banks, 1904) (Acarina: Tarsonemidae): A) The leves wrinkled for low e with petiole prolonged; B) wrinkling of the main ribbing (Photos: Bernardo A. Halfeld-Vieira)

\section{MATERIAL E MÉTODOS}

Em experimento realizado na casa de vegetação da Embrapa Roraima para testar a desempenho de alguns híbridos comercialmente utilizados pelos produtores do cinturão verde de Boa Vista, foi observado o ataque do ácaro-branco. Esse ataque foi controlado pelo uso do princípio ativo abamectina na dose de 1,8 g/100 1 d'água, aplicado em três pulverizações aos 40, 60 e 80 dias após o transplante. Recomenda-se observação contínua das plantas e aos primeiros sintomas da presença do ácaro-branco, folhas enrugadas para baixo e com pecíolo alongado, realizar a aplicação de acaricidas.

A avaliação da eficácia da abamectina no controle de $P$. latus foi realizada em três datas $(45,65$ e 85 dias após o transplante) quando foram quantificadas todas as plantas, por parcela, de cada um dos híbridos testados, baseando-se nos parâmetros: plantas atacadas (\%); plantas não atacadas (\%) e plantas recuperadas (\%) (plantas que deixaram de apresentar sintomas de ataque). Os dados referentes às taxas de plantas atacadas, não atacadas e recuperadas foram analisados segundo uma ANOVA com medidas repetidas no tempo (repeated measures ANOVA), sendo adotada a correção de probabilidade de Greenhouse-Geisser (G-G) (LITTEL et al., 1996). Os valores médios foram acrescidos dos respectivos erros-padrão e ordenados segundo o teste de comparação múltipla de Duncan, dada a significância do modelo. O nível de significância adotado, tanto para a ANOVA, quanto para os testes de comparação múltipla, foi de $5 \%(\alpha=0,05)$. As análises foram conduzidas com auxílio da proc glm e mixed do SAS ${ }^{\circledR}$ System e do pacote estatístico STATISTICA 5.5 (LITTEL et al., 1996, STATSOFT INC., 1999).

\section{RESULTADOS E DISCUSSÃO}

A Tabela 1 mostra que houve diferença estatística significativa entre os híbridos testados, quando comparados entre si ou em diferentes datas de avaliação. Já a interação híbridos x tempo não se mostrou significativa, uma vez que tanto o efeito temporal como o de híbridos são dependentes da aplicação do princípio ativo abamectina, mostrando que o controle de $P$. latus ocorreu de forma homogênea. 
TABELA 1 - Análise de variância para o modelo de medidas repetidas e significância do teste F a partir dos dados de controle de $P$. latus após pulverizações de abamectina aos 40, 60 e 80 dias após o transplante e obtidas em três avaliações.

Table 1 - Analysis of variance for the model of repeated measures and significance of $F$ test for the $P$. latus control data after sprayings of abamectina to the 40, 60 and 80 days after the transplant and obtained measures in three evaluations.

Onde: G-G-ajustedeprobabilidadede Greenhouse-Geisser; H-F-ajustedeprobabilidadedeHuynh-Feldt; ${ }^{*}$ diferençasignificativa $(\alpha=0,05) ; n$.s.: nãosignificativo

\begin{tabular}{|c|c|c|c|c|c|c|c|c|c|c|}
\hline & & \multirow[b]{2}{*}{ g.1. } & \multirow[b]{2}{*}{ QM } & \multicolumn{4}{|c|}{ Atacadas } & \multicolumn{3}{|c|}{ Sem ataque } \\
\hline & & & & $\mathrm{p}$ & $G-G$ & $\mathrm{H}-\mathrm{F}$ & QM & $\mathrm{p}$ & G-G & $\mathrm{H}-\mathrm{F}$ \\
\hline \multirow[t]{2}{*}{ Híbridos } & & 4 & 2188,26 & $* * *$ & & & 5083,40 & $* * * *$ & & \\
\hline & $\varepsilon 1$ & 29 & 390,21 & & & & 757,54 & & & \\
\hline Tempo & & 2 & 3991,43 & $* * * *$ & $* * *$ & $* * *$ & 2760,66 & $* * * *$ & $* * * *$ & $* * *$ \\
\hline \multirow[t]{2}{*}{ Tempo*Híbridos } & & 8 & 208,15 & n.s. & n.s. & n.s. & 39,18 & n.s. & n.s. & n.s. \\
\hline & $\varepsilon 2$ & 58 & 253,25 & & & & 68,98 & & & \\
\hline
\end{tabular}

A Figura 3 mostra o percentual de plantas atacadas por P. latus em avaliações aos 45, 65 e 85 dias após o transplante, onde se observa que o híbrido Nathalie apresentou o menor percentual de plantas atacadas em todas as avaliações, com uma variação de 13 a 28\% das plantas. O híbrido que apresentou o maior percentual de plantas atacadas foi Martha, com uma variação de 38 a 59\% das plantas (TABELA 2).

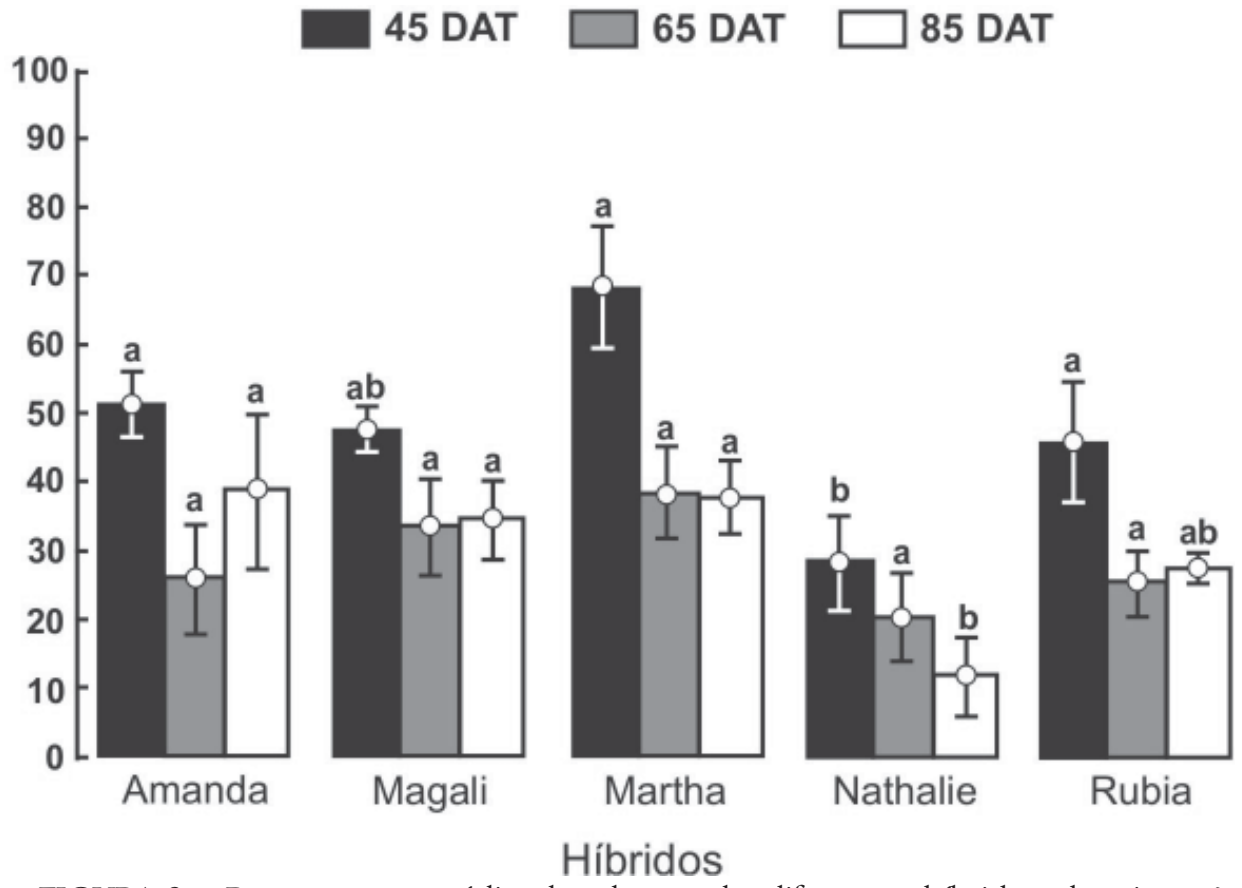

FIGURA 3 - Porcentagem média de plantas de diferentes hỉbridos de pimentão atacados pelo ácaro-branco Polyphagotarsonemus latus (Acari: Tarsonemidae) após pulverizações de abamectina aos 40, 60 e 80 dias após o transplante e obtidas em três avaliações. DAT: dias após o transplante; barras acompanhadas pelas mesmas letras não diferem significativamente entre si pelo teste de Duncan $(\alpha=0,05)$.

Figure 3 - Averagepercentage ofplants of differentsweetpepper hybrids attacked by the Polyphagotarsonemus latus mite-white (Acari: Tarsonemidae) aftersprayings of abamectina to the 40, 60 and 80 days after the transplant and obtained in three evaluations. DAT: days after the transplant; bars followed for the same letters do not differ significantly $\alpha=0.05$ ). 
TABELA 2 - Valores médios e desvio padrão do percentual de plantas atacadas (At.), não atacadas (NAt.) e recuperadas (Re.) dos híbridos avaliados, ao longo do experimento, ordenados segundo o teste de

Duncan $(\alpha=0,05)$, a partir dos dados de controle de $P$. latus após pulverizações de abamectina aos 40, 60 e 80 dias após o transplante (DAT) e obtidas em três avaliações. Boa Vista, RR, 2003.

Table 2 - Average values and standard deviation of the attacked plants percentage (At.), not attacked (NAt.) and recouped (Re.) of the hybrids evaluated, throughout the experiment, ordinate according to Duncan test $(\alpha=0.05)$, from the P. latus control data, after sprayings of abamectina to the 40,60 and 80 days after the transplant (DAT) and obtained in three evaluations.

Valores precedidos de mesma letra, na vertical, não diferem significativamente, segundo o teste de Duncan, a 5\% de significância.

\begin{tabular}{|c|c|c|c|c|c|c|c|c|}
\hline \multicolumn{4}{|c|}{45 DAT } & \multicolumn{3}{|l|}{65 DAT } & \multicolumn{2}{|l|}{85 DAT } \\
\hline Híbridos & At. & NAt. & At. & NAt. & Re. & At. & NAt. & Re. \\
\hline Amanda & $51,2 \pm 12,2 \mathrm{a}$ & $48,8 \pm 12,2 \mathrm{~b}$ & $26,0 \pm 19,5$ a & $33,4 \pm 18,8 \mathrm{~b}$ & $40,6 \pm 9,0 \quad a b$ & $38,7 \pm 27,6$ a & $38,9 \pm 20,3 b$ & $22,4 \pm 13,2 \mathrm{~b}$ \\
\hline Magali & $47,6 \pm 8,7$ & $52,4 \pm 8,7 \quad a b$ & $33,6 \pm 18,5$ a & $29,5 \pm 10 \quad b$ & $36,9 \pm 13,6 \mathrm{ab}$ & $34,4 \pm 15,1$ a & $37,5 \pm 11,3 \mathrm{~b}$ & $28,1 \pm 11,1 \mathrm{ab}$ \\
\hline Martha & $68,3 \pm 23,6$ a & $31,7 \pm 23,6 \mathrm{~b}$ & $38,6 \pm 17,3$ a & $10,0 \pm 9,2 \quad \mathrm{c}$ & $51,4 \pm 19,7$ a & $38,0 \pm 13,7$ a & $17,8 \pm 14,7 \mathrm{c}$ & $44,3 \pm 15,4$ a \\
\hline Nathalie & $28,3 \pm 17,7 \quad b$ & $71,7 \pm 17,7 \mathrm{a}$ & $20,6 \pm 16,6$ a & $54,2 \pm 19,3$ a & $25,2 \pm 12,0 \quad b$ & $12,2 \pm 13,4 \quad b$ & $62,8 \pm 18,4$ a & $25,0 \pm 16,6 \mathrm{~b}$ \\
\hline Rúbia & $45,9 \pm 24,7 \mathrm{ab}$ & $54,1 \pm 24,7 \mathrm{ab}$ & $25,3 \pm 12,6$ a & $42,0 \pm 19,8 \mathrm{ab}$ & $32,6 \pm 19,5 \mathrm{~b}$ & $27,5 \pm 5,5 \quad a b$ & $47,0 \pm 19,5 \mathrm{ab}$ & $25,5 \pm 20,7 \mathrm{~b}$ \\
\hline Total & $48,2 \pm 21,8$ & $51,8 \pm 21,8$ & $28,9 \pm 17,2$ & $33,9 \pm 21,3$ & $37,2 \pm 17,1$ & $29,9 \pm 18,2$ & $40,9 \pm 22$ & $29,2 \pm 16,8$ \\
\hline
\end{tabular}

As informações contidas na Figura 3 e Tabela 2 proporcionam maior segurança na escolha de cultivares, possibilitando que o produtor defina qual a cultivar mais adequada para o plantio. Caso o problema fitossanitário mais significativo na região de cultivo seja o ácaro-branco, o híbrido Nathalie é o mais recomendado para plantio, por ter apresentado o maior percentual de plantas não atacadas nas três avaliações. Da mesma maneira que o uso do híbrido Martha deve ser descartado por apresentar o maior percentual de plantas atacadas nas três avaliações.

A Figura 4 mostra o percentual de plantas recuperadas do ataque por $P$. latus (plantas que deixaram de apresentar sintomas) em avaliações aos 45, 65 e 85 dias após o transplante, onde se observa que o híbrido Martha apresentou o maior percentual de plantas recuperadas em todas as avaliações, com uma variação de 20 a 44\% das plantas. O híbrido que apresentou o menor percentual de plantas recuperadas foi Nathalie com uma variação de 9 a $24 \%$ das plantas conforme Tabela 2.

O parâmetro plantas recuperadas foi utilizado para caracterizar o efeito do tratamento com abamectina, tendo como base a redução no número de plantas atacadas após a aplicação do princípio ativo e pela observação de que as plantas recuperadas não sofreram ataque posterior ao tratamento. Desta maneira, o percentual de plantas recuperadas, observado com mais evidência no híbrido Martha, é decorrente do efeito do acaricida, não devendo ser considerado como uma característica dos híbridos. Entretanto, recomenda-se o estudo mais detalhado destas cultivares, a fim de avaliar se a resposta delas ao ataque de P.latus está associada com tolerância ou mesmo resistência a essa praga.

Pode-se observar neste trabalho que houve o aparecimento de novas plantas atacadas, mesmo após o uso do acaricida, entretanto, de forma reduzida. Essa observação pode ser confirmada pelo trabalho de diversos pesquisadores que estudando o efeito de acaricidas no controle de P. latus, nas culturas do pimentão e algodão, observaram a maior eficiência de abamectina, embora em percentuais de mortalidade não superiores a 90\% (MISRA, 2003; SRINIVASULU et al. 2002; BELLETTINI et al., 1999). Gavioli et al. (1988), além de comprovarem a eficiência de abamectina contra a referida praga, registram que esse mostrou boa seletividade contra os predadores Frankliniella schultzei e Leucothrips sp. 


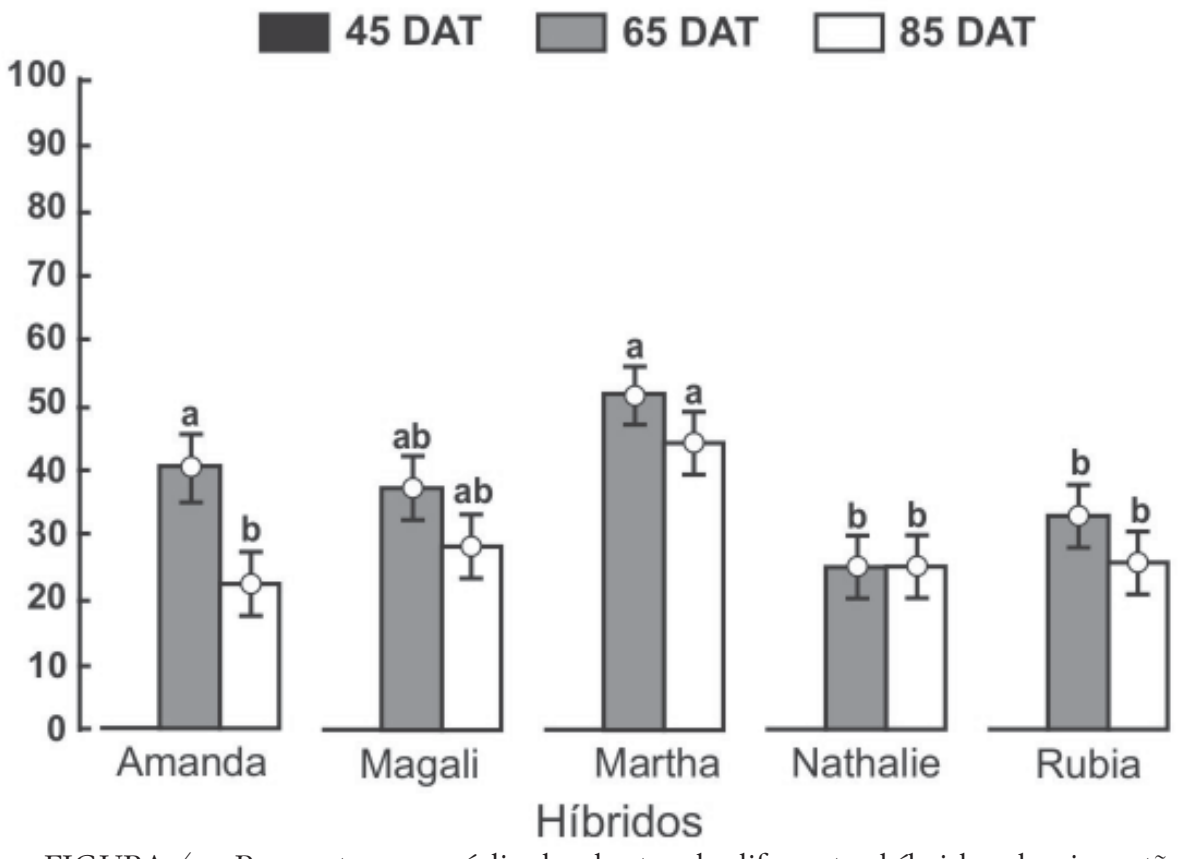

FIGURA 4 - Porcentagem média de plantas de diferentes híbridos de pimentão recuperados do ataque pelo ácaro-branco Polyphagotarsonemus latus (Acari: Tarsonemidae) após pulverizações de abamectina aos 40, 60 e 80 dias após o transplante e obtidas em três avaliações. DAT: dias após o transplante; barras acompanhadas pelas mesmas letras não diferem significativamente entre si pelo teste de Duncan $(\alpha=0,05)$.

Figure 4 - Average percentage of plants of different sweet pepper bybrids recouped of the attack of the mite-white Polyphagotarsonemus latus (Acari: Tarsonemidae) after sprayings of abamectina to the 40, 60 and 80 days after the transplant and obtained in three evaluations. DAT: days after the transplant; bars followed by the same letters do not differ significantly according to the Duncan test $(\alpha=0.05)$.

\section{CONCLUSÕES}

Os resultados justificam a escolha de cultivares como um importante componente do manejo do ácaro-branco, uma vez que a principal alternativa de controle químico, mesmo apresentando boa seletividade aos inimigos naturais, não reduz totalmente a população de P. latus.

\section{REFERÊNCIAS}

BELLETTINI, S.; ULBRICH, A. V.; GASTALDI, L. F. Controle do ácaro-branco Polyphagotarsonemus latus (Banks, 1904) com diferentes doses de clorfenapyr na cultura do algodoeiro. In: CONGRESSO BRASILEIRO DE ALGODÃO: O ALGODÃO NO SÉCULO XX, PERSPECTIVAS PARA O SÉCULO XXI, 2., 1999, Ribeirão Preto. Anais... Ribeirão Preto, 5-10 set. 1999. p. 152-154.

FLECHTMANN, C. H. W. Ácaros de importância agrícola. 7. ed. São Paulo: Nobel, 2000.

FNP. Agrianual 2003 Anuário da Agricultura Brasileira: melancia, São Paulo: FNP Consultoria e Informativos, 2003. $544 \mathrm{p}$.

GAllO, D. ET AL. Entomologia Agrícola. Piracicaba: FEALQ, 2002. 902 p. 
GAVIOLI, L. A. et al. Efeito de abamectina, cyfluthrin e fenpropathrin sobre os ácaros Polyphagotarsonemus latus (Banks 1904) e Tetranychus urticae (Koch 1836), e alguns inimigos naturais no algodoeiro. Ecossistema, v. 12, p. 66-77, 1987, 1988.

LITTEL, R. C. et al. SAS® System for Mixed Models. SAS Institute Inc. Cary, p. 633, 1996.

LORENTZ, L. H. et al. Estimativa da amostragem para pimentão em casa de vegetação plástica. Horticultura Brasileira, v. 20, n. 2, jul. 2002. Suplemento 2.

MISRA, H. P. Efficacy of newer insecticides in chilli leaf-curl management. Indian-Journal of Agricultural Sciences, v. 73, n. 6, p. 358-360, 2003.

NANNETI, D. C.; GOMES, L. A.; ELEOTÉRIO, R. Avaliação de genótipos de pimentão em cultivo orgânico dentro de ambiente protegido. Horticultura Brasileira, v. 20, n. 2, jul. 2002. Suplemento 2.

NASCIMENTO, I. R. et al. Avaliação de características produtivas de híbridos de pimentão. Horticultura Brasileira, v. 20, n. 2, jul. 2002. Suplemento 2.

OLIVEIRA, J. M. F.; LUZ, F. J. F. Orientação para cultivo do pimentão em Roraima. Embrapa Roraima: Circular Técnica, n. 2, nov. 1998, 24p.

SERRANO CERMEÑO, Z. Controle da atmosfera da casa de vegetação. In: SERRANO CERMEÑO, Z. Casa de vegetações: instalação e manejo. Lisboa: Liteza, p. 238-301, 1990.

SOROKER, V., et al. Whitefly wax as a cue for phoresy in the broad mite Polyphagotarsonemus latus (Acari: Tarsonemidae). Chemoecology, v. 13, n. 4, p. 163-168, 2003.

SRINIVASULU P.; NAIDU, V. G.; RAO, N. V. Evaluation of different pesticides for the control of yellow mite, Polyphagotarsonemus latus (Banks) on chilli. Journal of Applied Zoological Researches, v. 13, n. 1, p. 71-72, 2002.

STATSOFT, INC. STATISTICA for Windows. [Computer program manual]. Tulsa, OK: StatSoft, Inc. Disponível em: <http://www.statsoft.com>. Acesso em: 26 de ago. 1999. 